\section{Prevalência e fatores associados ao excesso de peso em adultos: inquérito populacional em Rio Branco, Acre, Brasil, 2007-2008}

\author{
Prevalence of overweight and associated factors \\ in adults: a population survey in Rio Branco, \\ Acre State, Brazil, 2007-2008
}

\footnotetext{
1 Centro de Ciências da Saúde e do Desporto, Universidade Federal do Acre, Rio Branco, Brasil.

\section{Correspondência} M. Z. R. Lino

Centro de Ciências da Saúde e do Desporto, Universidade Federal do Acre. Campus Universitário Reitor Áulio Gélio Alves de Souza, Rodovia BR 364, Km 04, 6637, Rio Branco, AC 69915-900, Brasil.

marinazago@gmail.com
}

\begin{abstract}
Population studies in Brazil have shown an increased prevalence of overweight and obesity in adult populations in all regions of the country. The objective was to estimate prevalence and identify risk factors associated with overweight among adults in Rio Branco, Acre State, Brazil. The study included a cross-sectional population-based sample of 1,469 adults. Multivariate analysis was performed using Poisson regression, taking overweight $\left(B M I \geq 25 \mathrm{~kg} / \mathrm{m}^{2}\right)$ as the dependent variable. Prevalence of overweight was 46.9\%, higher among women. Overweight tended to increase with age in both men and women. Overweight was associated with socioeconomic, demographic, and morbidity variables such as hypertension and dyslipidemia. The high prevalence of overweight in adults of both sexes suggests a public health problem. Control measures and prevention of health risks associated with excess weight are necessary.
\end{abstract}

Overweight; Obesity; Adult

\author{
Marina Zago Ramos Lino 1 \\ Pascoal Torres Muniz 1 \\ Kamile Santos Siqueira 1
}

\section{Introdução}

O excesso de peso corporal é um nível do estado nutricional caracterizado pelo peso corporal excessivo, derivado do acúmulo de gordura, em relação à altura, que pode propiciar maior risco para o desenvolvimento de diversas doenças. É oriundo do cálculo de um indicador, denominado índice de massa corporal (IMC), muito usado para a classificação do estado nutricional na população de adultos, por meio da sua categorização em pontos de corte 1 .

A obesidade é o estado mais grave do excesso de peso, sendo caracterizada como uma das doenças que integra o grupo de doenças e agravos não transmissíveis. A partir dos anos 60 , as doenças e agravos não transmissíveis ocuparam a principal posição entre as causas de mortes no Brasil 2. Estudos têm observado que indivíduos obesos apresentaram maior risco de desenvolver outras doenças como hipertensão arterial, enfermidades cardiovasculares, diabetes mellitus, dislipidemias, doenças gastrintestinais, osteoartrites, apneia do sono, distúrbios psicossociais, entre outras enfermidades 3,4 , e até mesmo de diminuição da expectativa de vida 5 . O desenvolvimento do excesso de peso e obesidade envolve múltiplos fatores, observados, principalmente, com as mudanças comportamentais do século $\mathrm{XX}$, tais como hábitos de vida, consumo alimentar, características socioambientais, susceptibilidade genética e biológica 1 . 
A Organização Mundial da Saúde (OMS) estimou, em 2005, que, globalmente, cerca de 1,6 bilhões de adultos (15 anos ou mais de idade) apresentavam excesso de peso, e, pelo menos, 400 milhões apresentavam obesidade. Projeções futuras da OMS estimam que, em 2015, aproximadamente, 2,3 bilhões de adultos estarão em condição de excesso de peso, e mais de 700 milhões estarão obesos 6 .

Estudos na Europa 7 e nos Estados Unidos 8,9 têm observado um crescimento da prevalência de excesso de peso e obesidade. Nos países em desenvolvimento, a tendência de aumento do excesso de peso corporal pode ser tratada como um reflexo do desenvolvimento de países desenvolvidos 10 , observado por pesquisas que apresentam dados de aumento da prevalência em alguns países da América Latina 11,12.

No Brasil, observa-se a tendência de aumento da prevalência de sobrepeso e obesidade como uma das características marcantes do processo de transição nutricional do país 13,14,15. Resultados comparativos de estudos na população brasileira entre 1974 e 2003 apresentam dados de crescimento da prevalência de excesso de peso e obesidade em adultos para todas as regiões. Especificamente na Região Norte do país, observa-se, a partir das principais pesquisas populacionais de âmbito nacional, uma tendência de aumento dessas prevalências, mesmo em regiões menos desenvolvidas do Brasil, apesar de essa tendência ter se mostrado temporalmente postergada quando comparada às grandes capitais e aos centros urbanos. Entretanto, pesquisas recentes têm observado resultados semelhantes entre a Região Norte e as demais regiões do país, apesar da escassez de pesquisas na região $16,17,18$.

Portanto, diante desse fato, adicionado aos aspectos de transição nutricional, que se observa no Brasil e no mundo, e à escassez de estudos na Região Norte, é importante avaliar o perfil de indivíduos com excesso de peso e os fatores associados na população residente na região. $\mathrm{O}$ presente estudo objetiva estimar a prevalência e identificar os principais fatores associados ao excesso de peso em adultos no Município de Rio Branco, Acre.

\section{Metodologia}

\section{Delineamento, local e amostra do estudo}

Trata-se de um estudo transversal de base populacional, parte integrante da pesquisa Saúde e Nutrição em Crianças e Adultos no Município de Rio Branco, Acre, realizado pela Universidade
Federal do Acre em parceria com a Secretaria de Vigilância em Saúde do Ministério da Saúde, Secretaria Estadual de Saúde do Acre e Secretaria Municipal de Saúde do Município de Rio Branco. A população de estudo foi composta de adultos acima de 18 anos de idade residentes no Município de Rio Branco.

Utilizou-se, comounidade primária amostral, a divisão do Município de Rio Branco, realizada pelo Instituto Brasileiro de Geografia e Estatística (IBGE; http://www.ibge.gov.br) para o Censo Demográfico 2000, em 250 setores censitários. Foi realizada amostra probabilística, complexa, em duas etapas, utilizando-se os seguintes procedimentos: na primeira etapa, foram sorteados 35 setores censitários, sendo 31 em área urbana e quatro em área rural, esses setores foram anteriormente sorteados para a Pesquisa Nacional por Amostra de Domicílios (PNAD) de 2006. Na segunda etapa, foram sorteados 25 domicílios por setor, totalizando 875 domicílios. O cálculo do tamanho da amostra esteve dentro dos critérios aceitáveis de precisão, como mostra a Tabela 1, com estimativa de 1.500 entrevistas. Para valores de prevalências estimadas para a população de maiores de 18 anos residentes em domicílios particulares permanentes no Município de Rio Branco, verificaram-se margens de erro (d) não superiores a 5\% e coeficientes de variação inferiores a $10 \%$, obtidos por meio da seguinte fórmula:

$$
C V(p)=\frac{\sqrt{\frac{\mathrm{p}}{\mathrm{n}} \mathrm{q}}}{\mathrm{p}} ;(\mathrm{d})=(1,96 \times \mathrm{xepa})
$$

Para suprir as prováveis perdas e recusas, foram escolhidos $15 \%$ de domicílios acima do necessário, elevando a amostra para 977 domicílios. Todos os adultos residentes nos domicílios participantes foram convidados a participar da pesquisa. A amostra selecionada foi composta por 1.516 indivíduos. Desse conjunto, foram excluídos das análises mulheres grávidas $(n=32)$ e sujeitos que não tiveram medidas aferidas de peso ou estatura ( $n=15$ ), com uma perda de $3,2 \%$, resultando em uma amostra final de 1.469 indivíduos.

\section{Coleta de dados}

O período de coleta do inquérito supracitado foi realizado entre novembro de 2007 e outubro de 2008. Os dados foram coletados por entrevistadores (enfermeiros, nutricionistas e estagiários) treinados pela coordenação da pesquisa.

O instrumento de coleta de dados utilizado foi um questionário individual elaborado com base nos questionários aplicados no Inquérito Vigilância de Fatores de Risco e Proteção para Doenças 
Erros-padrão, coeficiente de variação e margem de erro (d) [para $n=1.500$ e deff = 2].

\begin{tabular}{cccc}
\hline $\mathbf{p}$ & Erro-padrão & $\mathbf{d}$ & Coeficiente de variação (p) \\
\hline 0,2 & 0,013038 & 0,025555 & 0,086923 \\
0,3 & 0,015811 & 0,03099 & 0,063246 \\
0,4 & 0,017889 & 0,035062 & 0,044721 \\
\hline
\end{tabular}

Coeficiente de variação: variabilidade da prevalência; $d$ : margem de erro da amostra; erro-padrão: medida de variabilidade da prevalência; p: prevalência.

Crônicas por Inquérito Telefônico (VIGITEL Brasil 2006) 19 e no Inquérito Domiciliar sobre Comportamentos de Risco e Morbidade Referida de Doenças e Agravos não Transmissíveis: Brasil, 15 Capitais e Distrito Federal, 2002-2003 17. O questionário é composto de módulos temáticos, com questões fechadas, semiabertas e abertas, envolvendo as seguintes seções: (1) sociodemográfica e econômica, com dados de gênero, idade, estado civil, renda, escolaridade, ocupação, entre outros; (2) avaliação do uso e cobertura de serviços de saúde; (3) avaliação do estado de saúde individual, com dados de morbidade referida, estilo de vida, consumo de tabaco e álcool, atividade física, entre outros, e (4) avaliação do estado nutricional, com dados de antropometria e hábitos de consumo alimentar.

Os entrevistadores foram devidamente treinados para a coleta dos dados de antropometria e aferiram o peso e a estatura em duplicata no momento da entrevista. O peso foi obtido pela utilização de balança microeletrônica portátil (modelo BF572 Body Fat, Tanita Corp., Tóquio, Japão; capacidade de $130 \mathrm{~kg}$ com intervalos de $100 \mathrm{~g}$ ), e a estatura foi medida pelo estadiômetro portátil graduado em milímetros.

\section{Definição das variáveis}

Pelas medidas de peso e altura obtidas na coleta de dados, foi calculado o IMC de cada indivíduo por meio da divisão do peso (massa corporal em quilogramas) pelo quadrado da altura (em metros). Para caracterizar o estado nutricional da população do estudo, foram utilizados pontos de corte para o IMC, preconizados pela OMS 1, tendo sido o estado nutricional classificado como: baixo peso (IMC $<18,5 \mathrm{~kg} / \mathrm{m}^{2}$ ); eutrófico (IMC entre 18,5-24,9kg/m²); excesso de peso (IMC $\geq 25,0 \mathrm{~kg} / \mathrm{m}^{2}$ ), podendo esse último ser classificado como sobrepeso (IMC entre $25,0-29,9 \mathrm{~kg} / \mathrm{m}^{2}$ ) e obesidade (IMC $\geq 30,0 \mathrm{~kg} / \mathrm{m}^{2}$ ), entre outras classificações. Cada indivíduo foi classificado como baixo peso, peso adequado e excesso de peso. $\mathrm{Na}$ análise de prevalências e fatores associados, foi utilizado, como desfecho, o excesso de peso.

As variáveis sociodemográficas e socioeconômicas incluídas no estudo foram estratificadas. A idade foi categorizada em seis estratos: 18-24, 25-34, 35-44, 45-54, 55-64 e 65 ou mais anos de idade. A variável escolaridade foi dividida em três categorias segundo anos de estudo: 0-8, 9-11 e 12 ou mais. A variável renda foi obtida pela resposta referida pelo entrevistado informando a sua renda pessoal em salários mínimos e foi estratificada em quatro categorias: sem renda ou menor que um salário mínimo (SM), de 1-3 SM, 3-10 SM e 10 ou mais SM, com base no salário em vigor nos anos em que foram realizadas as entrevistas (entre R\$ 380,00 em 2007 e R\$ 415,00 em 2008). A ocupação foi tratada de acordo com a atividade referida pelo entrevistado, como empregado, aposentado ou afastado ou pensionista, do lar, estudante e desempregado. A situação conjugal foi dividida em duas categorias: solteiro, separado ou viúvo e casado ou em união estável.

Nas variáveis de hábitos de vida, o tabagismo foi categorizado, de acordo com o consumo referido, em: não fuma, fuma ocasionalmente, fuma diariamente e ex-fumante; o consumo de álcool foi estratificado de acordo com a quantidade de dias da semana que o entrevistado declarou beber: não consome ou menos de uma vez por semana, de 1-2 vezes por semana e 3 ou mais vezes por semana.

A variável relacionada à atividade física foi avaliada segundo a categorização preconizada pela OMS 20, sendo considerado ativo suficiente de lazer aqueles que se enquadram nos seguintes critérios: (1) indivíduos que praticam, pelo menos, 30 minutos diários de atividade física, de intensidade leve ou moderada, em cinco ou mais dias da semana; ou (2) indivíduos que praticam, pelo menos, 20 minutos diários de atividade física de intensidade vigorosa, em três ou mais dias da semana. Foram classificados como práticas 
de intensidade leve ou moderada as atividades como: caminhada, caminhada em esteira, musculação, hidroginástica, ginástica em geral, natação, artes marciais, ciclismo e voleibol; corrida, corrida em esteira, ginástica aeróbica, futebol, basquetebol e tênis foram classificados como práticas de intensidade vigorosa. A variável inatividade física foi construída com referência na mesma utilizada pelo VIGITEL Brasil 2006 19, caracterizada e categorizada como indivíduos em condição de completa inatividade física aqueles que informam que: (1) não praticaram qualquer atividade física no lazer nos últimos três meses; (2) não realizavam esforços físicos intensos no trabalho (não andavam muito, não carregavam peso e não faziam outras atividades equivalentes em termos de esforço físico); (3) não se deslocavam para o trabalho a pé ou de bicicleta; e (4) não eram responsáveis pela limpeza pesada de suas casas.

A percepção de saúde autorreferida foi dicotomizada apresentado as categorias: excelente/ boa e regular/ruim. As variáveis relacionadas a morbidades, como hipertensão, diabetes e dislipidemias, foram avaliadas por questões que perguntavam se algum médico informou se apresentou a respectiva morbidade. Tais questões requereram respostas dicotomizadas em “sim" ou "não”.

Em relação aos hábitos dietéticos, a variável utilizada no estudo foi relacionada à dieta para perder peso, na qual o sujeito da pesquisa referiu estar ou não realizando algum tipo de dieta no momento da entrevista. Das variáveis referentes ao questionário de frequência alimentar, utilizou-se consumo de frutas e consumo de vegetais. $\mathrm{O}$ consumo de frutas na amostra foi categorizado de forma que indivíduos que relataram consumir, pelo menos, uma vez ao dia um tipo de fruta foram considerados "consumidor diário”, os demais indivíduos que responderam consumir menos de sete dias na semana foram incluídos como "não consumidor diário". O consumo de vegetais seguiu a mesma categorização das frutas, na qual indivíduos que responderam que consomem, pelo menos, um tipo de legume cozido ou salada crua todos os dias se enquadram em "consumidor diário" de vegetais, os demais sujeitos que declararam consumir menos de uma vez ao dia foram enquadrados em "não consumidor diário". As referidas categorias foram preferidas na tentativa de aproximar a categorização da variável ao preconizado pela OMS, que é de, no mínimo, 400g (cinco porções de 80g) de frutas e vegetais ao dia, classificados como A e B 22; e diante do fato de o questionário aplicado no inquérito não possibilitar a mensuração de quantidades diárias.

\section{Análise dos dados}

O cálculo de amostra e banco de dados foi realizado através do programa estatístico Epi Info 6.04 (Centers for Disease Control and Prevention, Atlanta, Estados Unidos). Para a análise estatística, foi utilizado o programa Stata/SE 10.0 (Stata Corp., College Station, Estados Unidos). Todas as análises foram realizadas utilizando o comando survey para possibilitar a expansão da amostra realizada por conglomerados.

A análise dos dados foi realizada em etapas, sendo sempre categorizadas segundo o gênero. Primeiramente, foi realizada a estatística descritiva, com cálculos de distribuição e caracterização da amostra segundo variáveis de interesse do estudo. Em seguida, foram estimadas prevalências de excesso de peso, segundo as variáveis independentes. A etapa posterior envolveu análise bivariada entre o desfecho excesso de peso e as variáveis independentes selecionadas no estudo, com obtenção de estimativas de risco baseadas em razões de prevalência brutas e ajustadas por faixa etária, através de regressão de Poisson, utilizando-se nível de significância de 0,05 e intervalo de 95\% de confiança (IC95\%).

As variáveis independentes foram trabalhadas de forma que um estrato era tratado como referência, e as estimativas de risco dos demais estratos eram obtidas em relação a esse de referência.

Para identificar os fatores associados ao desfecho e estabelecer o controle de variáveis, intervenientes e de confusão, foi construído um modelo multivariado, utilizando a regressão de Poisson, sendo selecionadas, como candidatas ao modelo, as covariáveis que apresentaram significância inferior a $20 \%$ (valor de p < 0,20), e mantiveram-se, no modelo final, as variáveis com significância estatística (IC95\%, desconsiderando valor de nulidade) após ajuste.

\section{Procedimentos éticos}

O projeto de pesquisa foi aprovado pelo Comitê de Ética em Pesquisa da Universidade Federal do Acre. Todos os investigados foram informados quanto à participação voluntária na pesquisa, sendo esclarecidos os objetivos e processos da pesquisa, e assinaram um Termo de Consentimento Livre e Esclarecido.

\section{Resultados}

A amostra do presente estudo foi composta por 1.469 indivíduos, desses, 45,3\% eram homens, e $54,7 \%$, mulheres. A distribuição das caracterís- 
ticas sociodemográficas e socioeconômicas da população total, categorizadas segundo o gênero, está representada na Tabela 2 . A amostra foi distribuída em seis estratos de faixa etária, em que se observa maior proporção de adultos nas faixas etárias de 18-24 anos (24\%) e 25-34 anos $(24,1 \%)$. Observou-se a predominância de indivíduos solteiros, separados ou viúvos; moradores de zona urbana; que possuem atividade remunerada; indivíduos de escolaridade entre 0-8 anos de estudo; e pessoas que referiram não possuir renda ou possuir renda menor que um SM.

Quanto ao estado nutricional, a amostra apresentou $46,9 \%$ de indivíduos com excesso de peso, subdivididos em $31 \%$ com sobrepeso e $15,9 \%$ com obesidade. De acordo com o gê- nero, a distribuição do estado nutricional para excesso de peso apresentou-se maior nas mulheres $(51,8 \%)$ do que nos homens $(41 \%)$, como demonstra a Figura 1.

$\mathrm{Na}$ análise descritiva das prevalências de excesso de peso na população total e segundo o gênero, observaram-se maiores prevalências em homens nas faixas etárias de 35-44 anos e 55-64 anos, nos indivíduos do estrato de renda mais elevado, de zona urbana, sem companheira, que declaram possuir trabalho remunerado e maior escolaridade (12 ou mais anos de estudo). Segundo morbidades autorreferidas, homens que referiram possuir hipertensão arterial e dislipidemias apresentaram maiores prevalências de excesso de peso, observadas também naqueles

Tabela 2

Distribuição das características sociodemográficas e socioeconômicas da população adulta acima de 18 anos segundo o sexo. Município de Rio Branco, Acre, Brasil, 2007-2008.

\begin{tabular}{|c|c|c|c|c|c|c|}
\hline \multirow[t]{2}{*}{ Variáveis } & \multicolumn{2}{|c|}{ Total } & \multicolumn{2}{|c|}{ Masculino } & \multicolumn{2}{|c|}{ Feminino } \\
\hline & $N(1.469)$ & $\%$ & n (649) & $\%$ & n (820) & $\%$ \\
\hline \multicolumn{7}{|l|}{ Faixa etária (anos) } \\
\hline $18-24$ & 355 & 24,0 & 178 & 26,8 & 177 & 22,0 \\
\hline $25-34$ & 364 & 24,1 & 160 & 24,0 & 204 & 24,3 \\
\hline $35-44$ & 294 & 18,5 & 125 & 18,4 & 169 & 18,8 \\
\hline $45-54$ & 194 & 12,6 & 77 & 9,6 & 117 & 14,5 \\
\hline $55-64$ & 147 & 12,1 & 60 & 12,2 & 87 & 12,2 \\
\hline 65 ou mais & 115 & 8,7 & 49 & 9,0 & 66 & 8,2 \\
\hline \multicolumn{7}{|l|}{ Estado conjugal } \\
\hline Casado/União estável & 656 & 43,0 & 259 & 40,5 & 397 & 45,0 \\
\hline Solteiro/Separado/Viúvo & 813 & 57,0 & 390 & 59,5 & 423 & 55,0 \\
\hline \multicolumn{7}{|l|}{ Ocupação } \\
\hline Trabalho remunerado & 820 & 54,6 & 440 & 69,1 & 380 & 42,7 \\
\hline Estudante & 87 & 5,1 & 42 & 5,2 & 45 & 5,0 \\
\hline Desempregado & 212 & 13,8 & 87 & 11,8 & 125 & 15,4 \\
\hline Do lar & 167 & 12,3 & - & - & 167 & 22,6 \\
\hline Aposentado/Pensionista/Afastado & 183 & 14,2 & 80 & 13,9 & 103 & 14,3 \\
\hline \multicolumn{7}{|l|}{ Renda $(\mathrm{SM}) *$} \\
\hline Não possui ou < 1 & 566 & 42,4 & 168 & 30,2 & 398 & 52,2 \\
\hline $1-3$ & 619 & 41,6 & 326 & 49,3 & 293 & 35,4 \\
\hline $3-10$ & 205 & 13,1 & 112 & 16,7 & 93 & 10,2 \\
\hline 10 ou mais & 49 & 2,9 & 28 & 3,8 & 21 & 2,2 \\
\hline \multicolumn{7}{|l|}{ Escolaridade (anos de estudo) } \\
\hline $0-8$ & 680 & 52,1 & 313 & 54,6 & 367 & 50,0 \\
\hline $9-11$ & 543 & 33,1 & 236 & 31,1 & 307 & 34,7 \\
\hline 12 ou mais & 240 & 14,8 & 100 & 14,3 & 140 & 15,3 \\
\hline \multicolumn{7}{|l|}{ Zona de moradia } \\
\hline Rural & 116 & 28,4 & 59 & 31,7 & 57 & 25,6 \\
\hline Urbana & 1.353 & 71,6 & 590 & 68,3 & 763 & 74,4 \\
\hline
\end{tabular}

* Renda obtida baseada no salário mínimo (SM) do Brasil no período de 2007-2008, valores entre R\$380,00 e R\$415,00. 
Distribuição do estado nutricional de adultos de acordo com o índice de massa corporal (IMC) segundo sexo. Rio Branco, Acre, Brasil, 2007-2008.

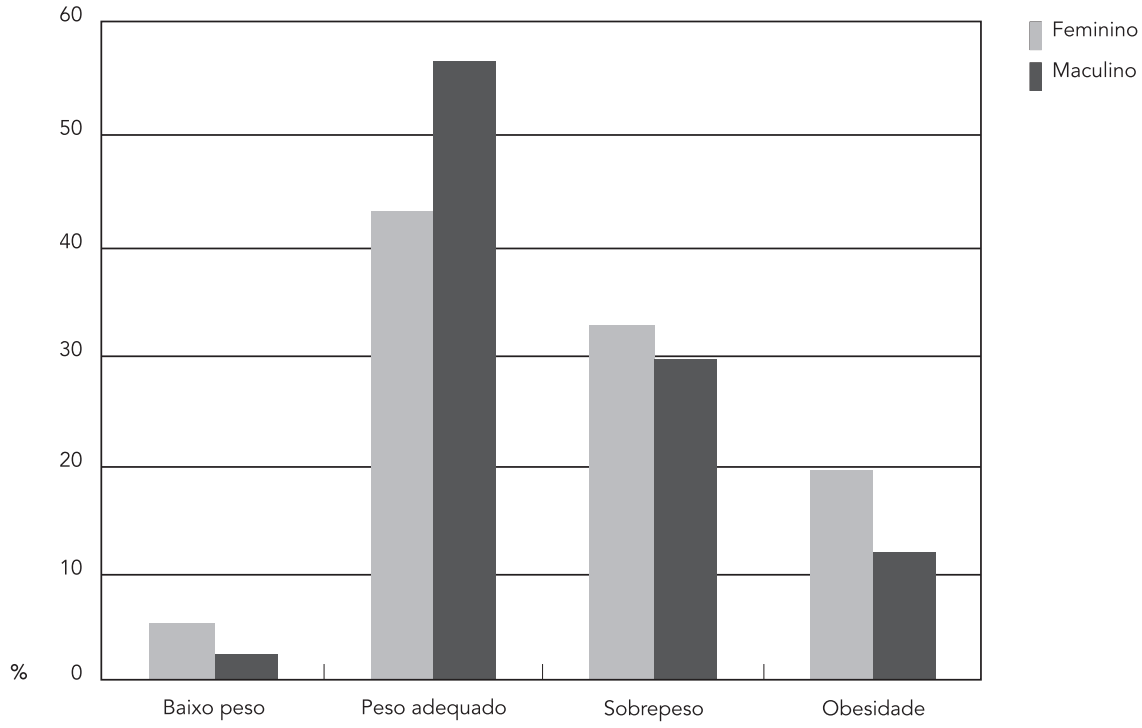

que a autopercepção de saúde foi considerada regular/ruim (Tabela 3). Homens que declaram estar em dieta e ex-fumantes tiveram maiores prevalências de excesso de peso. Em mulheres, resultados semelhantes aos homens foram observados, sendo o excesso de peso mais prevalente na faixa etária de 55-64 anos e nas mulheres de renda de 10 ou mais SM. Resultados discrepantes com o sexo masculino foram observados em mulheres que tiveram suas prevalências de excesso de peso mais elevadas em relação à zona de moradia (rural) e à escolaridade (0-8 anos de estudo). As mulheres com maiores prevalências de excesso de peso foram as que referiram morbidades hipertensão arterial e dislipidemias, bem como percepção de saúde regular/ruim, adesão de dieta para perder peso e que declararam ser ex-fumantes (Tabela 3).

As razões de prevalência (RP) brutas entre as variáveis independentes e a variável desfecho excesso de peso segundo o gênero são apresentadas na Tabela 4. Para os adultos do sexo masculino, observaram-se prevalências de excesso de peso $259 \%$ maiores nas faixas etárias entre 35 44 anos (IC95\%: 2,49-5,18), e, no sexo feminino, a prevalência se apresentou $197 \%$ mais elevada no estrato entre 55-64 anos (IC95\%: 1,74-5,07), em relação ao estrato de 18-24 anos, sendo que, em ambos os gêneros, o teste de tendência linear apresentou significância estatística $(\mathrm{p}<0,001)$ para aumento do excesso de peso com o aumento da idade.

Nas estimativas de risco, foram encontradas maiores prevalências estatisticamente significantes nos homens: casados ou em união estável ( $\mathrm{RP}=1,88$; IC95\%: 1,49-2,37) em relação aos solteiros, separados e viúvos; moradores de zona rural (RP = 1,85; IC95\%: 1,24-2,76) em relação aos de zona urbana; com renda de 10 ou mais SM $(\mathrm{RP}=3,02$; IC95\%: 2,06-4,43) em relação à menor faixa de renda, em que o teste de tendência linear foi significativo para aumento de excesso de peso com o aumento da renda ( $\mathrm{p}<0,001)$; nos ex-fumantes $(\mathrm{RP}=1,54$; IC95\%: 1,17-2,02) em relação aos fumantes; nos que autorreferem hipertensão arterial (RP = 1,44; IC95\%: 1,19-1,74) e dislipidemias ( $R P=1,94$; IC95\%: 1,52-2,47); nos que referem estar em dieta para perder peso $(\mathrm{RP}=1,92$; IC95\%: 1,60-2,31) e consumo diário de frutas (RP = 1,34; IC95\%: 1,15-1,56), segundo demonstra a Tabela 4 .

Nas mulheres da amostra, as maiores prevalências de excesso de peso foram encontradas nas que autorreferem hipertensão arterial ( $\mathrm{RP}=$ 1,72; IC95\%: 1,54-1,92) e dislipidemias (RP = 1,67; IC95\%: 1,39-2,01) em relação àquelas que 
Prevalências de excesso de peso (índice de massa corporal - IMC $\geq 25 \mathrm{~kg} / \mathrm{m}^{2}$ ) em adultos segundo sexo entre as variáveis do estudo. Rio Branco, Acre, Brasil, 2007-2008.

\begin{tabular}{|c|c|c|c|c|c|c|c|c|c|}
\hline \multirow[t]{3}{*}{ Variáveis } & \multicolumn{9}{|c|}{ Excesso de peso } \\
\hline & \multicolumn{3}{|c|}{ Total } & \multicolumn{3}{|c|}{ Masculino } & \multicolumn{3}{|c|}{ Feminino } \\
\hline & N (1.469) & $\%$ & Valor de $p$ & n (649) & $\%$ & Valor de $p$ & n (820) & $\%$ & Valor de $p$ \\
\hline \multicolumn{10}{|l|}{ Faixa etária (anos) } \\
\hline $18-24$ & 355 & 21,6 & & 178 & 17,7 & & 177 & 25,6 & \\
\hline $25-34$ & 364 & 44,4 & & 160 & 44,0 & & 204 & 44,7 & \\
\hline $35-44$ & 294 & 63,8 & & 125 & 63,6 & & 169 & 63,9 & \\
\hline $45-54$ & 194 & 59,5 & & 77 & 59,1 & & 117 & 59,8 & \\
\hline $55-64$ & 147 & 61,2 & & 60 & 42,7 & & 87 & 76,5 & \\
\hline 65 ou mais & 115 & 49,3 & $<0,001$ & 49 & 33,6 & $<0,001$ & 66 & 63,5 & 0,009 \\
\hline \multicolumn{10}{|l|}{ Estado conjugal } \\
\hline Solteiro/Separado/Viúvo & 656 & 39,1 & & 259 & 26,8 & & 397 & 48,3 & \\
\hline Casado/União estável & 813 & 52,6 & $<0,001$ & 390 & 50,5 & $<0,001$ & 423 & 54,5 & 0,029 \\
\hline \multicolumn{10}{|l|}{ Ocupação } \\
\hline Trabalho remunerado & 820 & 47,7 & & 440 & 42,9 & & 380 & 54,1 & \\
\hline Estudante & 87 & 21,8 & & 42 & 29,4 & & 45 & 15,2 & \\
\hline Desempregado & 212 & 38,4 & & 87 & 35,2 & & 125 & 40,4 & \\
\hline Do lar & 167 & 51,0 & & - & - & & 167 & 51,0 & \\
\hline Aposentado/Pensionista/Afastado & 183 & 57,1 & $<0,001$ & 80 & 40,3 & 0,123 & 103 & 70,4 & $<0,001$ \\
\hline \multicolumn{10}{|l|}{ Renda $(\mathrm{SM}) *$} \\
\hline Não possui ou < 1 & 566 & 39,0 & & 168 & 26,3 & & 398 & 44,9 & \\
\hline $1-3$ & 619 & 50,2 & & 326 & 43,3 & & 293 & 58,0 & \\
\hline $3-10$ & 205 & 55,6 & & 112 & 54,6 & & 93 & 56,9 & \\
\hline 10 ou mais & 49 & 76,2 & $<0,001$ & 28 & 79,6 & $<0,001$ & 21 & 71,5 & 0,004 \\
\hline \multicolumn{10}{|l|}{ Escolaridade (anos de estudo) } \\
\hline $0-8$ & 680 & 50,6 & & 313 & 40,3 & & 367 & 60,0 & \\
\hline $9-11$ & 543 & 40,8 & & 236 & 38,7 & & 307 & 42,4 & \\
\hline 12 ou mais & 240 & 47,3 & 0,009 & 100 & 47,9 & 0,399 & 140 & 46,8 & 0,004 \\
\hline \multicolumn{10}{|l|}{ Zona de moradia } \\
\hline Rural & 116 & 39,0 & & 59 & 25,8 & & 57 & 51,4 & \\
\hline Urbana & 1353 & 49,9 & $<0,001$ & 590 & 47,9 & $<0,001$ & 763 & 52,5 & 0,889 \\
\hline \multicolumn{10}{|l|}{ Consumo de tabaco } \\
\hline Não fumante & 1.203 & 44,6 & & 352 & 37,9 & & 483 & 49,5 & \\
\hline Fuma ocasionalmente & 47 & 40,5 & & 24 & 30,8 & & 23 & 51,4 & \\
\hline Fuma diariamente & 219 & 35,4 & & 110 & 26,2 & & 109 & 45,3 & \\
\hline Ex-fumante & 368 & 59,4 & $<0,001$ & 163 & 58,5 & $<0,001$ & 205 & 60,2 & 0,107 \\
\hline \multicolumn{10}{|l|}{ Consumo de álcool (dia/semana) } \\
\hline Não consome ou < 1 & 1.237 & 47,2 & & 484 & 39,4 & & 753 & 52,4 & \\
\hline $1-2$ & 201 & 44,6 & & 141 & 44,2 & & 60 & 45,4 & \\
\hline 3 ou mais & 31 & 47,6 & 0,842 & 24 & 52,6 & 0,318 & 7 & 28,5 & 0,343 \\
\hline \multicolumn{10}{|l|}{ Percepção de saúde autorreferida } \\
\hline Excelente/Boa & 852 & 41,1 & & 422 & 35,4 & & 430 & 47,3 & \\
\hline Regular/Ruim & 615 & 54,0 & $<0,001$ & 227 & 50,8 & 0,006 & 388 & 55,8 & 0,006 \\
\hline \multicolumn{10}{|l|}{ Hipertensão autorreferida } \\
\hline Não & 1.073 & 39,6 & & 501 & 36,8 & & 572 & 42,1 & \\
\hline Sim & 396 & 64,9 & $<0,001$ & 148 & 53,2 & 0,001 & 248 & 72,5 & $<0,001$ \\
\hline
\end{tabular}

(continua) 


\begin{tabular}{|c|c|c|c|c|c|c|c|c|c|}
\hline \multirow[t]{3}{*}{ Variáveis } & \multicolumn{9}{|c|}{ Excesso de peso } \\
\hline & \multicolumn{3}{|c|}{ Total } & \multicolumn{3}{|c|}{ Masculino } & \multicolumn{3}{|c|}{ Feminino } \\
\hline & $N(1.469)$ & $\%$ & Valor de $p$ & n (649) & $\%$ & Valor de $p$ & n (820) & $\%$ & Valor de p \\
\hline \multicolumn{10}{|c|}{ Diabetes autorreferida } \\
\hline Não & 1.400 & 46,1 & & 618 & 40,1 & & 782 & 51,1 & \\
\hline Sim & 69 & 61,1 & 0,030 & 31 & 55,7 & 0,079 & 38 & 66,9 & 0,129 \\
\hline \multicolumn{10}{|c|}{ Dislipidemias autorreferidas } \\
\hline Não & 1.220 & 41,1 & & 567 & 36,3 & & 653 & 45,4 & \\
\hline Sim & 249 & 74,2 & $<0,001$ & 82 & 70,5 & $<0,001$ & 167 & 76,2 & $<0,001$ \\
\hline \multicolumn{10}{|c|}{ Estar em dieta para perder peso } \\
\hline Não & 1.291 & 42,9 & & 606 & 38,8 & & 685 & 46,7 & \\
\hline Sim & 178 & 79,1 & $<0,001$ & 43 & 74,7 & $<0,001$ & 135 & 80,5 & $<0,001$ \\
\hline \multicolumn{10}{|c|}{ Atividade física suficiente no lazer } \\
\hline $\operatorname{Sim}$ & 245 & 44,9 & & 136 & 39,2 & & 109 & 52,2 & \\
\hline Não & 1.224 & 47,2 & 0,629 & 513 & 41,3 & 0,763 & 711 & 51,6 & 0,911 \\
\hline \multicolumn{10}{|c|}{ Condição de inatividade física } \\
\hline Não & 1.375 & 46,3 & & 590 & 39,4 & & 785 & 51,8 & \\
\hline Sim & 94 & 54,2 & 0,307 & 59 & 55,8 & 0,057 & 35 & 50,8 & 0,914 \\
\hline \multicolumn{10}{|c|}{ Consumo de frutas (porção diária) } \\
\hline Menos de 1 & 1.133 & 43,5 & & 528 & 38,7 & & 605 & 47,9 & \\
\hline 1 & 336 & 58,8 & $<0,001$ & 121 & 51,9 & $<0,001$ & 215 & 62,4 & 0,003 \\
\hline \multicolumn{10}{|c|}{ Consumo de legumes (porção diária) } \\
\hline Menos de 1 & 994 & 44,5 & & 470 & 38,9 & & 524 & 49,9 & \\
\hline 1 & 475 & 52,4 & 0,014 & 179 & 47,3 & 0,079 & 296 & 55,4 & 0,168 \\
\hline
\end{tabular}

* Renda obtida baseada no salário mínimo (SM) do Brasil no período de 2007-2008, valores entre $\mathrm{R} \$ 380,00$ e $\mathrm{R} \$ 415,00$.

não referem tais morbidades. Nas variáveis de hábitos alimentares, observou-se prevalência $30 \%$ maior de excesso de peso entre as mulheres que referem consumir frutas diariamente (IC95\%: 1,12-1,50) em relação às que não consomem diariamente; e prevalência $72 \%$ maior entre as que referem estar em dieta para perder peso (IC95\%: 1,50-1,97), comparado às que não estão em dieta (Tabela 4).

A análise multivariada de regressão de Poisson para os fatores associados ao excesso de peso segundo gênero tem seus modelos resultantes da análise ajustada apresentados na Tabela 4. No modelo para o sexo masculino das variáveis testadas como fatores associados ao excesso de peso, as que permaneceram significativas foram faixa etária, hipertensão arterial autorreferida, dislipidemias autorreferidas, ex-fumantes, estar em dieta para perder peso e consumo diário de frutas. Nas mulheres, os fatores que se associaram ao excesso de peso no modelo foram faixa etária, consumo de tabaco referido diariamente, hipertensão arterial autorreferida, dislipidemias autorreferidas e estar em dieta para perder peso.

\section{Discussão}

O presente estudo apresentou resultados, obtidos a partir de amostra probabilística, que evidenciam altas prevalências de excesso de peso em adultos de ambos os sexos, constituindo um problema de saúde pública no Município de Rio Branco. O IMC foi utilizado como indicador do estado nutricional da população estudada e, apesar de não indicar alguns aspectos da composição corporal do indivíduo, é um bom indicador e tem sido utilizado em estudos epidemiológicos de delineamento transversal devido à facilidade de sua mensuração, à grande disponibilidade de dados de massa corporal e estatura e à relação com morbimortalidade comprovada na literatura 22.

Na Região Norte do país, o inquérito INCA de 2002-2003 17, realizado em 15 capitais da federação e no Distrito Federal, apresentou dados das cidades de Manaus (Amazonas) e Belém (Pará), onde, nessas capitais, as prevalências de excesso de peso na população acima de 15 anos de idade foram de 39\% e 35,1\%, respectivamente. Segundo dados do VIGITEL Brasil 2008 18, realizado por meio de entrevistas telefônicas, a prevalência de 
Razões de prevalência (RP) brutas e ajustadas segundo o modelo multivariado de fatores associados ao excesso de peso (índice de massa corporal - IMC $\geq$ $25 \mathrm{~kg} / \mathrm{m}^{2}$ ) em adultos segundo sexo entre as variáveis do estudo. Rio Branco, Acre, Brasil, 2007-2008.

\begin{tabular}{|c|c|c|c|c|}
\hline \multirow[t]{3}{*}{ Variáveis } & \multicolumn{4}{|c|}{ Excesso de peso } \\
\hline & \multicolumn{2}{|c|}{ Masculino } & \multicolumn{2}{|c|}{ Feminino } \\
\hline & RP bruta (IC95\%) & RP ajustada * (IC95\%) & RP bruta (IC95\%) & RP ajustada * (IC95\%) \\
\hline \multicolumn{5}{|l|}{ Faixa etária (anos) } \\
\hline $18-24$ & 1,00 & 1,00 & 1,00 & 1,00 \\
\hline $25-34$ & $2,48(1,58-3,89)$ & $2,31(1,42-3,77)$ & $1,74(0,72-4,19)$ & $1,71(0,79-3,73)$ \\
\hline $35-44$ & $3,59(2,49-5,18)$ & $2,45(1,74-3,44)$ & $2,48(1,26-4,88)$ & $2,30(1,18-4,46)$ \\
\hline $45-54$ & $3,34(1,93-5,75)$ & $2,13(1,42-2,74)$ & $2,32(1,04-5,19)$ & $2,03(0,93-4,41)$ \\
\hline $55-64$ & $2,41(1,43-4,05)$ & $1,76(1,13-2,74)$ & $2,97(1,74-5,07)$ & $2,38(1,35-4,18)$ \\
\hline 65 ou mais & $1,89(1,25-2,87)$ & $1,26(0,81-1,97)$ & $2,47(0,97-6,27)$ & $1,95(0,79-4,82)$ \\
\hline Valor de $p$ de tendência linear & $<0,001$ & $<0,001$ & $<0,001$ & $<0,001$ \\
\hline \multicolumn{5}{|l|}{ Estado conjugal } \\
\hline Solteiro/Separado/Viúvo & 1,00 & 1,00 & 1,00 & 1,00 \\
\hline Casado/União estável & $1,88(1,49-2,37)$ & $1,20(0,94-1,53)$ & $1,12(1,01-1,25)$ & $1,09(0,89-1,33)$ \\
\hline \multicolumn{5}{|l|}{ Ocupação } \\
\hline Trabalha & 1,00 & & 1,00 & \\
\hline Estudante & $0,68(0,38-1,22)$ & & $0,28(0,14-0,55)$ & \\
\hline Desempregado & $0,82(0,53-1,26)$ & & $0,74(0,61-0,91)$ & \\
\hline Do lar & - & & $0,94(0,82-1,07)$ & \\
\hline Aposentado/Pensionista/Afastado & $0,94(0,69-1,27)$ & & $1,30(1,09-1,54)$ & \\
\hline \multicolumn{5}{|l|}{ Renda $(\mathrm{SM}) \star \star$} \\
\hline Não possui ou < 1 & 1,00 & 1,00 & 1,00 & 1,00 \\
\hline $1-3$ & $1,64(1,16-2,32)$ & $1,13(0,82-1,55)$ & $1,29(1,08-1,53)$ & $1,08(0,92-1,26)$ \\
\hline $3-10$ & $2,07(1,57-2,73)$ & $1,12(0,86-1,46)$ & $1,26(1,04-1,52)$ & $0,96(0,81-1,14)$ \\
\hline 10 ou mais & $3,02(2,06-4,43)$ & $1,33(0,98-1,82)$ & $1,59(1,10-2,28)$ & $1,12(0,78-1,62)$ \\
\hline Valor de $p$ de tendência linear & $<0,001$ & $<0,001$ & $<0,001$ & $<0,001$ \\
\hline \multicolumn{5}{|l|}{ Escolaridade (anos de estudo) } \\
\hline $0-8$ & 1,00 & & 1,00 & \\
\hline $9-11$ & $0,96(0,77-1,18)$ & & $0,70(0,57-0,87)$ & \\
\hline 12 ou mais & $1,18(0,86-1,63)$ & & $0,78(0,59-1,03)$ & \\
\hline Valor de $p$ de tendência linear & 0,707 & & $<0,001$ & \\
\hline \multicolumn{5}{|l|}{ Zona de moradia } \\
\hline Rural & 1,00 & 1,00 & 1,00 & \\
\hline Urbana & $1,85(1,24-2,76)$ & $1,77(1,33-2,36)$ & $0,97(0,72-1,32)$ & \\
\hline \multicolumn{5}{|l|}{ Consumo de tabaco } \\
\hline Não fumante & 1,00 & 1,00 & 1,00 & 1,00 \\
\hline Fuma ocasionalmente & $0,81(0,36-1,82)$ & $0,94(0,36-2,45)$ & $1,03(0,63-1,68)$ & $1,00(0,61-1,63)$ \\
\hline Fuma diariamente & $0,69(0,39-1,21)$ & $0,67(0,41-1,08)$ & $0,91(0,72-1,15)$ & $0,80(0,65-0,98)$ \\
\hline Ex-fumante & $1,54(1,17-2,02)$ & $1,37(1,02-1,85)$ & $1,21(0,99-1,47)$ & $0,91(0,78-1,07)$ \\
\hline \multicolumn{5}{|l|}{ Consumo de álcool (dia/semana) } \\
\hline Não consome ou < 1 & 1,00 & & 1,00 & \\
\hline $1-2$ & $1,21(0,91-1,37)$ & & $0,86(0,61-1,21)$ & \\
\hline 3 ou mais & $1,33(0,85-2,08)$ & & $0,54(0,15-1,92)$ & \\
\hline \multicolumn{5}{|l|}{ Percepção de saúde autorreferida } \\
\hline Excelente/Boa & 1,00 & 1,00 & 1,00 & 1,00 \\
\hline Regular/Ruim & $1,43(1,08-1,90)$ & $1,20(0,99-1,45)$ & $1,17(1,04-1,32)$ & $0,97(0,81-1,15)$ \\
\hline
\end{tabular}

(continua) 


\begin{tabular}{|c|c|c|c|c|}
\hline \multirow[t]{3}{*}{ Variáveis } & \multicolumn{4}{|c|}{ Excesso de peso } \\
\hline & \multicolumn{2}{|c|}{ Masculino } & \multicolumn{2}{|c|}{ Feminino } \\
\hline & RP bruta (IC95\%) & RP ajustada * (IC95\%) & RP bruta (IC95\%) & RP ajustada * (IC95\%) \\
\hline \multicolumn{5}{|c|}{ Hipertensão autorreferida } \\
\hline Não & 1,00 & 1,00 & 1,00 & 1,00 \\
\hline Sim & $1,44(1,19-1,74)$ & $1,22(1,05-1,42)$ & $1,72(1,54-1,92)$ & $1,42(1,18-1,70)$ \\
\hline \multicolumn{5}{|c|}{ Diabetes autorreferida } \\
\hline Não & 1,00 & & 1,00 & \\
\hline Sim & $1,38(0,98-1,96)$ & & $1,30(0,97-1,76)$ & \\
\hline \multicolumn{5}{|c|}{ Dislipidemias autorreferidas } \\
\hline Não & 1,00 & 1,00 & 1,00 & 1,00 \\
\hline $\operatorname{Sim}$ & $1,94(1,52-2,47)$ & $1,25(0,93-1,68)$ & $1,67(1,39-2,01)$ & $1,22(1,03-1,45)$ \\
\hline \multicolumn{5}{|l|}{ Estar em dieta } \\
\hline Não & 1,00 & 1,00 & 1,00 & 1,00 \\
\hline Sim & $1,92(1,60-2,31)$ & $1,26(0,96-1,65)$ & $1,72(1,50-1,97)$ & $1,53(1,34-1,75)$ \\
\hline \multicolumn{5}{|c|}{ Atividade física suficiente no lazer } \\
\hline $\operatorname{Sim}$ & 1,00 & & 1,00 & \\
\hline Não & $1,05(0,74-1,49)$ & & $0,98(0,81-1,19)$ & \\
\hline \multicolumn{5}{|c|}{ Condição de inatividade física } \\
\hline Não & 1,00 & & 1,00 & \\
\hline Sim & $1,41(1,05-1,90)$ & & $0,98(0,68-1,40)$ & \\
\hline \multicolumn{5}{|c|}{ Consumo de frutas (porção diária) } \\
\hline Menos de 1 & 1,00 & 1,00 & 1,00 & 1,00 \\
\hline 1 & $1,34(1,15-1,56)$ & $1,19(1,02-1,39)$ & $1,30(1,12-1,50)$ & $1,10(0,96-1,27)$ \\
\hline \multicolumn{5}{|c|}{ Consumo de vegetais (porção diária) } \\
\hline Menos de 1 & 1,00 & & 1,00 & \\
\hline 1 & $1,21(0,98-1,49)$ & & $1,11(0,95-1,29)$ & \\
\hline
\end{tabular}

* Variáveis ajustadas no sexo masculino por faixa etária, estado conjugal, renda, zona de moradia, tabagismo, percepção de saúde, hipertensão arterial, dislipidemias, adoção de dieta e consumo de frutas. Variáveis ajustadas no sexo feminino por faixa etária, estado conjugal, renda, tabagismo, percepção de saúde, hipertensão arterial, dislipidemias, adoção de dieta e consumo de frutas;

** Renda obtida baseada no salário mínimo do Brasil no período de 2007-2008, valores entre R\$380,00 e R\$415,00.

excesso de peso, em Manaus, foi de $42,2 \%$ e, em Belém, de 46,4\%; porém, no Município de Rio Branco, a prevalência de excesso de peso apresentou resultados mais elevados, mesmo quando comparados às demais capitais da Região Norte, como Porto Velho (Rondônia), Boa Vista (Roraima), Macapá (Amapá) e Palmas (Tocantins).

A Pesquisa de Orçamentos Familiares (POF) 16 apresenta dados da Região Norte para excesso de peso segundo sexo, em 2003-2004. Segundo a POF, a prevalência de excesso de peso, na região, foi de $36,4 \%$ para o sexo masculino e $36 \%$ para o sexo feminino. Comparando as prevalências de excesso de peso segundo o sexo, observa-se maior prevalência, no Município de Rio Branco, em mulheres, $51,8 \%$, e, em homens, 45,3\%, resultado encontrado discordante do encontrado pelo VIGITEL Brasil 2008 18, que apresentou resultado oposto entre os sexos, com prevalência de excesso de peso de $39,6 \%$ em mulheres e 56,3\% em homens.
De acordo com dados da POF, o excesso de peso tende a aumentar com a idade, apresentando dados de $48,3 \%$ de excesso de peso na faixa etária de 35-44 anos, enquanto, no Município de Rio Branco, observou-se prevalência mais elevada, em torno de $63 \%$ de homens dessa faixa etária com excesso de peso. Em mulheres, a POF apresenta uma prevalência $57,4 \%$ na faixa de $55-64$ anos, os resultados do presente estudo mostram prevalências maiores nas mulheres do Município de Rio Branco, com 76,5\% de 55-64 anos 16.

Os resultados do presente estudo mostraram teste de tendência linear significante $(p<0,05)$, com aumento da prevalência de excesso de peso à medida que aumenta a faixa de idade em ambos os gêneros. Resultados semelhantes em relação à associação com a idade foram observados em diversos estudos de mesmo delineamento 10,16,23. Entretanto, não é possível estabelecer a relação temporal do fenômeno por ser 
um estudo seccional e, portanto, todas as observações foram feitas em cada indivíduo em uma única oportunidade.

Diversos estudos mostram associações entre situação socioeconômica e risco de excesso de peso e obesidade, com comportamentos diferentes entre homens e mulheres, e nas diferentes regiões, levando-se, em conta, fatores de hábitos de vida e de desenvolvimento que podem ser discrepantes de acordo com o local do estudo. Estudo sobre resultados da Pesquisa Sobre Padrões de Vida (PPV), nas regiões Nordeste e Sudeste do país, em 1996-1997, apresenta diferentes prevalências de obesidade entre homens e mulheres e suas respectivas associações com renda e escolaridade nessas regiões com diferentes níveis de desenvolvimento 24 .

O excesso de peso apresentou tendência linear $(\mathrm{p}<0,05)$ sugestiva de ser maior nos maiores valores de renda em ambos os sexos. No sexo masculino, a RP, ajustada para idade, apresenta duas vezes maior prevalência de excesso de peso em indivíduos com renda de 10 ou mais SM em relação aos de renda entre 0-1 SM, com intervalo de confiança significante, concordando com estudos que encontraram resultados de tendência semelhante 24,25,26. Em relação à escolaridade, a prevalência de excesso de peso em homens teve associação positiva com o aumento de anos de estudo, concordando com estudos na população brasileira 18,24 que mostraram maior prevalência de excesso de peso em indivíduos com 12 anos ou mais de estudo.

Nas mulheres, a prevalência de excesso de peso se apresentou $59 \%$ maior naquelas de renda de 10 ou mais SM em relação à categoria de referência de 0-1 SM, com intervalo de confiança significante na RP bruta. Dados semelhantes foram encontrados em estudos na cidade de Salvador (Bahia) 23 e na Região Nordeste 24 do país. Resultados discrepantes foram observados em Pelotas (Rio Grande do Sul) ${ }^{26}$ para obesidade e na Região Sudeste 24 do país, onde a tendência foi significante de menores prevalências de obesidade nas maiores faixas de renda em mulheres, concordando com a hipótese de comportamentos diferentes de associação entre renda e obesidade em regiões distintas do país. Essa diferença regional nas prevalências advém do processo de desenvolvimento regional no Brasil, pois esse comportamento do excesso de peso em relação à renda nos países desenvolvidos é recente e advindo da melhoria do desenvolvimento regional e consequente maiores níveis de escolaridade e maior preocupação da população com a saúde das mulheres. Inclusive, estudos apontam para tendência de menor prevalência de excesso de peso e obesidade nos maiores níveis de escola- ridade em mulheres 18,24,27 e sugerem que a tendência de menores prevalências de excesso de peso na população de maior renda será observada nas décadas seguintes também para a região Norte e Nordeste 24. Entretanto, por enquanto, no presente estudo, observou-se que, no sexo feminino, a maior prevalência de excesso de peso foi observada no estrato de menor escolaridade, porém sem significância estatística.

No modelo multivariado de fatores associados ao excesso de peso, algumas variáveis ajustadas, como, por exemplo, estar em dieta para perder peso no momento da entrevista, apresentaram-se associadas positivamente ao excesso de peso quando comparadas a indivíduos que não estavam em dieta. Condição semelhante foi observada em estudo na cidade de Salvador, entretanto, um cuidado na interpretação dessa informação é necessário em estudos transversais por não se saber a relação de temporalidade dos acontecimentos. Oliveira et al. 23 apontam que esse fenômeno pode ser explicado pelo fato de que, uma vez que o indivíduo se percebe acima do peso, o mesmo tende a tomar medidas de restrição para controlar o excesso de peso.

$\mathrm{O}$ excesso de peso se mostrou mais prevalente em indivíduos que relataram não praticar atividade física suficiente e também em indivíduos em condição de inatividade física, especialmente no sexo masculino. O consumo de frutas e vegetais diariamente também se mostrou associado ao excesso de peso. Semelhante estudo sobre obesidade, utilizando ponto de corte do IMC $\geq$ $30 \mathrm{~kg} / \mathrm{m}^{2}$, no Município de Pelotas 28 , apresentou associação com algumas variáveis de morbidades e hábitos de vida. Faltam informações mais detalhadas sobre as práticas alimentares e o padrão de atividade física no Brasil em associação a dados antropométricos para uma melhor explicação dessas relações 29 .

As morbidades referidas hipertensão arterial e dislipidemias se mostraram associadas ao excesso de peso, pois apresentaram razões de prevalência, em ambos os sexos, maiores do que nos indivíduos que não declararam essas morbidades presentes, sugerindo o risco de comorbidades e concordando com achados na literatura 1,4,30,31.

Estudos transversais podem ter sua interpretação dificultada em relação a variáveis relacionadas a morbidades, atividade física e hábitos dietéticos, entre outras, uma vez que existe a possibilidade de viés de causalidade reversa, que ocorre quando a aparente exposição é consequência do desfecho, como é referido também em estudos que obtiveram resultados que também encontraram essa possível relação 23,28. Diante da carência de estudos de amostra representativa da população na região e da consistên- 
cia dos achados com a literatura, as prevalências e associações apresentadas no presente estudo são de grande relevância. Sugere-se, portanto, outros estudos longitudinais para verificar a determinação das associações observadas.
Com base nos resultados apresentados, tornam-se necessárias medidas de controle e prevenção dos riscos à saúde associados ao excesso de peso, tendo, como pressuposto, ações de incentivo para hábitos de vida saudáveis.

\section{Resumo}

Estudos populacionais no Brasil apontam aumento da prevalência de excesso de peso e obesidade na população adulta, em todas as regiões. O objetivo deste estudo foi estimar a prevalência e identificar os principais fatores associados ao excesso de peso em adultos, em Rio Branco, Acre, Brasil, por meio de um estudo transversal de base populacional com amostra composta de 1.469 adultos. A análise multivariada foi realizada através de regressão de Poisson, adotando-se, como variável dependente, excesso de peso (IMC $\left.\geq 25 \mathrm{~kg} / \mathrm{m}^{2}\right)$. A prevalência de excesso de peso foi observada em 46,9\% da amostra, sendo mais elevada entre as mulheres. Obser- vou-se tendência de aumento do excesso de peso com o aumento da idade em ambos os gêneros. Observaramse associações entre o excesso de peso e variáveis socioeconômicas, demográficas e morbidades como hipertensão arterial e dislipidemias. A alta prevalência de excesso de peso em adultos de ambos os sexos sugere um problema de saúde pública. Tornam-se necessárias medidas de controle e prevenção dos riscos à saúde associados ao excesso de peso.

Sobrepeso; Obesidade; Adulto 


\section{Colaboradores}

M. Z. R. Lino participou da análise dos dados e redação do artigo. K. S. Siqueira e P. T. Muniz colaboraram na redação e revisão crítica do artigo.

\section{Referências}

1. World Health Organization. Obesity: preventing and managing the global epidemic. Geneva: World Health Organization; 2000. (Technical Report Series, 894).

2. Ministério da Saúde. Vigilância de doenças e agravos não transmissíveis. http://portal.saude.gov.br/ saude/visualizar_texto.cfm?idtxt=25341 (acessado em 20/Nov/2008).

3. Francischi RPP, Pereira LO, Freitas CS, Klopfer M, Santos RC, Vieira P, et al. Obesidade: atualização sobre sua etiologia, morbidade e tratamento. Rev Nutr 2000; 13:17-28.

4. Souza LJ, Gicovate Neto C, Chalita FEB, Reis AFF, Bastos DA, Souto Filho JTD, et al. Prevalência de obesidade e fatores de risco cardiovascular em Campos, Rio de Janeiro. Arq Bras Endocrinol Metab 2003; 47:669-76.

5. Fontaine KR, Redden DT, Wang C, Westfall AO, Allison DB. Years of life lost due to obesity. JAMA 2003; 289:187-93.

6. World Health Organization. Obesity and overweight. http://www.who.int/mediacentre/fact sheets/fs311/en/index.html (acessado em 17/ Out/2009).

\section{Agradecimentos}

A realização desta investigação foi viabilizada por meio da colaboração acadêmica estabelecida entre o Programa de Mestrado em Saúde Coletiva da Universidade Federal do Acre e do Programa de Pós-graduação em Saúde Pública e Meio Ambiente da Fundação Oswaldo Cruz a qual vem sendo apoiada com recursos do Conselho Nacional de Desenvolvimento Científico e Tecnológico (CNPq; Edital UFAC-Fiocruz, processo 620024/2008) e Ministério da Saúde. Ao CNPq (processo $136011 / 2008-0$ ) pelo apoio financeiro.
7. European Association for the Study of Obesity. Obesity in Europe, 3. Brussels: International Obesity Task Force; 2005.

8. Flegal KM, Carroll MD, Kuczmarski RJ, Johnson CL. Overweight and obesity in the United States: prevalence and trends, 1960-1994. Int J Obes Relat Metab Disord 1998; 22:39-47.

9. Ogden CL, Carroll MD, McDowell MA, Flegal KM. Obesity among adults in the United States - no statistically significant change since 2003-2004. Hyattsville: National Center for Health Statistics; 2007. (NCHS Data Brief, 1).

10. Popkin BM. The nutrition transition and obesity in the developing world. J Nutr 2001; 131:871S-3S.

11. Sánchez-Castillo CP, Velázquez-Monroy O, Berber A, Lara-Esqueda A, Tapia-Conyer R, James WP, et al. Anthropometric cutoff points for predicting chronic diseases in the Mexican National Health Survey 2000. Obes Res 2003; 11:442-51.

12. Kain J, Vio F, Albala C. Obesity trends and determinant factors in Latin America. Cad Saúde Pública 2003; 19 Suppl 1:S77-86. 
13. Monteiro CA, Benicio MHD'A, Conde WL, Popkin BM. Shifting obesity trends in Brazil. Eur J Clin Nutr 2000; 54:342-6.

14. Batista Filho M, Rissin A. A transição nutricional no Brasil: tendências regionais e temporais. Cad Saúde Pública 2003; 19 Suppl 1:S181-91.

15. Batista Filho M, Souza AI, Miglioli TC, Santos MC. Anemia e obesidade: um paradoxo da transição nutricional brasileira. Cad Saúde Pública 2008; 24 Suppl 2:S247-57.

16. Instituto Brasileiro de Geografia e Estatística. Pesquisa de Orçamentos Familiares 2002-2003: análise da disponibilidade domiciliar de alimentos e do estado nutricional no Brasil. Rio de Janeiro: Instituto Brasileiro de Geografia e Estatística; 2004.

17. Instituto Nacional de Câncer. Inquérito domiciliar sobre comportamentos de risco e morbidade referida de doenças e agravos não transmissíveis: Brasil, 15 capitais e Distrito Federal 2002-2003. Rio de Janeiro: Instituto Nacional de Câncer; 2004.

18. Secretaria de Vigilância em Saúde, Ministério da Saúde. VIGITEL Brasil 2008. Vigilância de fatores de risco e proteção para doenças crônicas por inquérito telefônico. Brasília: Ministério da Saúde; 2009.

19. Secretaria de Vigilância em Saúde, Ministério da Saúde. VIGITEL Brasil 2006. Vigilância de fatores de risco e proteção para doenças crônicas por inquérito telefônico. Brasília: Ministério da Saúde; 2007.

20. World Health Organization. Recommended amount of physical activity. http://www.who.int/ dietphysicalactivity/factsheet_recommendations/ en (acessado em 16/Out/2009).

21. World Health Organization. Report of a Joint WHO/FAO Expert Consultation. Diet, nutrition and the prevention of chronic diseases. Geneva: World Health Organization; 2003. (Technical Report Series, 916)

22. Anjos LA. Índice de massa corporal (massa corporal/ estatura $^{2}$ ) como indicador do estado nutricional de adultos: revisão da literatura. Rev Saúde Pública 1992; 26:431-6.
23. Oliveira LPM, Assis AM, Silva MCM, Santana MLP, Santos NS, Pinheiro SMC, et al. Fatores associados a excesso de peso e concentração de gordura abdominal em adultos na cidade de Salvador, Bahia, Brasil. Cad Saúde Pública 2009; 25:570-82.

24. Monteiro CA, Conde WL, Popkin BM. Independent effects of income and education on the risk of obesity in the Brazilian adult population. J Nutr 2001; 131:881S-6S.

25. Peixoto MRG, Benicio MHD'A, Jardim PCBV. The relationship between body mass index and lifestyle in a Brazilian adult population: a cross-sectional survey. Cad Saúde Pública 2007; 23:2694-740.

26. Gigante DP, Dias-da-Costa JS, Olinto MTA, Menezes AMB, Macedo S. Obesidade da população adulta de Pelotas, Rio Grande do Sul, Brasil, e associação com nível socioeconômico. Cad Saúde Pública 2006; 22:1873-9.

27. Velásquez-Meléndez G, Pimenta AM, Kac G. Epidemiologia do sobrepeso e da obesidade e seus fatores determinantes em Belo Horizonte (MG), Brasil: estudo transversal de base populacional. Rev Panam Salud Pública 2004; 16:308-14.

28. Gigante DP, Barros FC, Post LA, Olinto MTA. Prevalência de obesidade em adultos e seus fatores de risco. Rev Saúde Pública 1997; 31:236-46.

29. Mendonça CP, Anjos LA. Aspectos das práticas alimentares e da atividade física como determinantes do crescimento do sobrepeso/obesidade no Brasil. Cad Saúde Pública 2004; 20:698-709.

30. Sartorelli DS, Franco LJ. Tendências do diabetes mellitus no Brasil: o papel da transição nutricional. Cad Saúde Pública 2003; 19:S29-36.

31. Sarno F, Monteiro CA. Importância relativa do índice de massa corporal e da circunferência abdominal na predição da hipertensão arterial. Rev Saúde Pública 2007; 41:788-96.

Recebido em 30/Jun/2010

Versão final reapresentada em 13/Dez/2010 Aprovado em 24/Jan/2011 